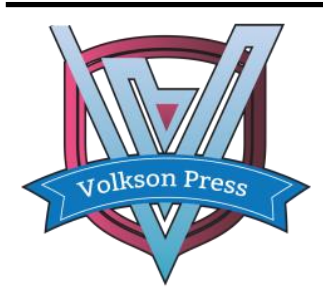

Contents List available at VOLKSON PRESS

Engineering \& Technology Innovations (ETI)

DOI : http://doi.org/10.26480/iceti.01.2017.86.89

\title{
ADVANCE OF INVERT SIMULATION METHODS FOR GROUNDWATER POLLUTION SOURCE IDENTIFICATION
}

\author{
Yuqiao Long1, 2, Tingting Cui', 2, Wei Li1,2, Zhongping Yang ${ }^{3}$,Yongwei Gai ${ }^{4}$ \\ 1. Nanjing Hydraulic Research Institute, Nanjing, China; \\ 2. State Key Laboratory of Hydrology-Water Resources and Hydraulic Engineering, Nanjing, China; \\ 3. College of civil engineering, Chongqing University, Chongqing, China; \\ 4. Water Resources Service Center of Jiangsu Province, Nanjing, China
}

This is an open access article distributed under the Creative Commons Attribution License, which permits unrestricted use, distribution, and reproduction in any medium, provided the original work is properly cited

\section{ARTICLE DETAILS}

\section{Article History:}

Received 02 october 2017 Accepted 06 october 2017

Available online 11 october 2017

Keywords:

groundwater; pollution; source identification; simulation.

\section{ABSTRACT}

Groundwater pollution source identification (GPSI) is an important support of contaminate removing, groundwater protecting, potable water security. As a typical ill-posed problem, GPSI has been studied for thirty years, and a brief review is given to conclude the characteristics of GPSI problems and the simulation method. The invert simulation method could be classified into four types: simulation-optimization method, analytical and regression method, direct method, and stochastic method. Most of these methods were studied with ideal numerical cases, further researches may focus on more complex GPSI problem, expressing physical chemistry and biological process, improving GPSI modeling efficiency, and model uncertainty.

\section{INTRODUCTION}

Groundwater is one of the crutial and irreplaceable resources for economic and social development ${ }^{[1]}$. In the recent twenty years, some China's plains have to endure the dense population, and intense human activities. Groundwater quality is getting worse because of the industrial and living sewage, pesticide, leakage of petroleum tank and landfill yard. According to the survey of shallow groundwater in 118 cities in China, $97.5 \%$ cities' groundwater was polluted, and $40 \%$ was serious polluted ${ }^{[2]}$. It is very important to protect groundwater so as to assure the sustainable development and survival safety of our descendants.

In order to protect groundwater, a lot of researches have been done in China. Most of these have been focused on the pollution-preventing policies, contaminate-cleaning approaches and technologies. However, the researches on identification of pollution source are rarely reported.

Pollution source identification (PSI) refers to reconstructing the pollution source locations and releasing histories from observed concentration records ${ }^{[3]}$. As one of the first steps in environmental remediation project, PSI can be classified into three typical types ${ }^{[4]}$ : namely, finding the release history of a source, finding the location of a source, and recovering the initial distribution of a contaminant plume. The PSI is helpful to making a cost-effective remediation strategy, partitioning the cleanup cost among liable parties ${ }^{[5]}$

Invert simulation methods for groundwater pollution source identification is one of the efficient approaches of PSI[4], and it has been extensively investigated in the past thirty years. At first, these methods were tested in 1D ideal problems ${ }^{[5,6]}$. Now they could be used to identify the pollution source in 2D heterogeneous arquifer ${ }^{[7,8]}$. Atmadja and Bagtzoglou (2010) ${ }^{[9]}$ have given a perfect report about these methods and subdivided the existing methods into four major groups, namely optimization, analytical and direct methods as well as probabilistic and geo-statistical approaches. However, due to the uncertainty of parameters of aquifer, pollution concentration observation and the simulation model, these simulation methods still could be used in some simple case, and some new method are proposed of PSI recently, so we give a further review of these methods.

\section{Characteristics of PS}

The PSI problems are inversing problems in nature. Forensic inversing problems can be generally divided in to the following two broad categories based on their mathematical treatments and potential applications: fullestimation problems and function-fitting problems ${ }^{[10]}$. Full-estimation problems describe forensic/inverse problems involving the determination of an entire timewise source history at a specific location or, conversely, an entire spatial distribution of some parameter at some given time. To define characteristics of this type of problems is that there is no known functional form for the distribution being sought. Such problems have been referred to as infinite dimensional minimization problems. Function-fitting problems describe problems which prior knowledge is available as to the form of the concentration or flux functions to be estimated. As the number of parameters to be estimated becomes larger, the conceptual distinction between function-fitting problems and full-estimation problems becomes less clear. PSI has some features as following:

It is difficult to solve the PSI models. Convection diffusion equation (CDE) is the basic control equation for contaminator migration. At present, no approaches can perfectly solve such problem which consists of the convection term and hyperbolic term (for convection) and the parabolic term (for diffusion). Therefore it is still a barrier for contamination modeling. Solving PSI requires solving the CDE backwards in time ${ }^{[11]}$. Such problems are typical ill-posed problems which are more difficult to solve than the pure CDE problems. Besides, diffusion process is irreversible, which make the PSI problems more complex.

PSI results heavily depend on the uncertainty of basic data. The effectiveness of PSI results can be sensitive to the observed data, and the number of inputs which are being estimated[12], and also could be influenced by hydrodynamic dispersion, model uncertainty, measurement error, and missing observed data ${ }^{[4]}$. Solution of the PSI is extremely sensitive to errors in the input data; even small observed error of contamination plume can disturb the estimation of the plume history ${ }^{[5]}$. Though researchers cannot ignore the uncertainty of boundary conditions, hydraulic conductivity, and diffusion coefficient[13], the sensitivity can be reduced by the algorithm used to solve the PSI issue. Sun, et al. (2006) ${ }^{[3]}$ 
used a constrained robust least squares to solve a PSI issue; the method could reduce the sensitivity induced by their technology.

PSI is a synthesized discipline. Solving PSI is not only related to the mathematical physics, stochastic, and optimization, it is also related to the chemistry and biology. More knowledge of different disciplines is required for solving PSI problems.

PSI is a very practical research field. PSI could be a substitute for field investigation and trials, and be economical for both time and money. It also can be taken as a foundation used to judge the responsibility of pollutionmakers. The PSI is a hotspot in theoretical research, and it is also an urgent practical work for pollution control.

\section{Invert simulation methods}

\subsection{Optimization approach}

Solution methods for PSI could be usually divided into non-heuristic and heuristic algorithm. The non-heuristic algorithms contain linear program $[14]$, non-linear program ${ }^{[15]}$, quadratic programming[3], and Augmented Lagrange Multiplier Method (ALMM) ${ }^{[16]}$. The algorithms are limited by the derivability and continuity of the PSI problems, and could not fit all the requirement of the PSI.

The heuristic algorithm, such as genetic algorithm, simulated annealing, particle swarm algorithm, are used in the PSI. These algorithms are suitable to the non-linear and non-convex optimization problems. We just introduce some common methods here.

The genetic algorithm (GA) is the most common solution method for PSI. Aral, et al (2001) ${ }^{[17]}$ apply the GA on the PSI of a 2D aquifer. To enhance the global search ability, the GA combined with perturbation theory ${ }^{[18]}$ is applied on a field investigation in Zibo. However, because the local search ability is weak, a GA-LS approach is proposed and applied on a 3D PSI problem. The GA is better than the artificial neural networks (ANNs), when there are several sources in a field. The convergence speed and solution accuracy of GA is depended on its parameters which are not easy to be decided. GA is time-consuming, when it is applied on the large scale groundwater problem with a lot of decision variants.

Tabu Search (TS) is a global optimization algorithm. Simulated annealing (SA) is a probabilistic metaheuristic for the global optimization problem of locating a good approximation to the global optimum of a given function in a large search space. It is often used when the search space is discrete. Yeh, et al (2007) propose the TASA approach ${ }^{[19]}$. The TS decides the source locations from the suspected locations, and the SA calculates the trial value of the release concentration and time. The approach less depends on the priori of the source location. At least, six observed points (each point has four observed values) are needed to estimate the correct release history. However, the TASA is applied on the constant release condition; it needs to be test under a dynamic release condition.

Mirghani, et al (2009) ${ }^{[12]}$ apply the evolutionary strategy on a 3D theoretical case. They find that the heterogeneity of the aquifer has no effect on the search efficiency of the algorithm. When more decision variants are used to estimate the characteristics of the pollution source, the optimization problem becomes more complex, and the nonconsistency between the prediction error and the solving error becomes more notable.

Bharat, et al. (2009) ${ }^{[20]}$ use particle swarm algorithm (PSA) to reconstruct the plum history in 1D groundwater flow. They find that the pollutant release curve is sharp, and need to be smoothed by the global constraints. In order to take account of the global and local optimum, SQUADS method which consist of PSA and a Levenberg-Marquardt optimization strategy is proposed by Vesselinov and Harp (2010) [21]. This method is very efficient and can obtain a good consistency between the estimation results and the observed data.

In the optimization approach, the forward simulation model will be run many times and the each solution will be compared to the observed data. So it will cost huge computation effort. At present, code parallelization ${ }^{[12]}$ and surrogate model[22] are two common approaches to reduce the computation effort.

Code parallelization is an efficient way to solve the complex PSI problem quickly. Mirghani, et al. (2009) ${ }^{[12]}$ point out that the calculation speed is one hundred times faster than the approach without code parallelization. However, the code parallelization require that the researchers must be familiar with source code, which is a barrier for widely use.
Surrogate model is based on the ANN. A simulation model is used to train the ANN. Then the trained ANN model substitutes the simulation model in the optimization approach. Singh and Datta point out that the ANN is an extension of optimum statistic pattern method [22]. They apply the ANN on the 2D theoretical PSI problem, and propose the index to evaluate the model efficiency and construction of ANN. The effects of the ANN are studied in problem with/without observed error and missing observed error. Though it could solve these problems, it still has some limit: the ANN training decides the effect of the PSI result; the aquifer is supposed to be homogenous.

\subsection{Analytical approach}

Analytical approach is more calculation efficient than the optimization approach, and is suitable for the PSI problem of inert pollutant in the simple hydrogeological condition. Ala and Domenico (1992) ${ }^{[23]}$ identified the quantity and scope of the pollution source in the Otis Air Force Base. The pollutants are chloride, boron, trichloroethylene and tetrachloroethylene. Butcher and Gauthier (1994) ${ }^{[24]}$ analyzed the DNAPL in an industrial site in the northeast of Massachusetts. They could proof the existence of the DNAPL source, but could not identify the source volume. Sidauruk, et al (1998)[25] evaluated the dispersion coefficient, initial location of the source in a $2 \mathrm{D}$ homogeneous aquifer with simple geometry.

Alapate and Kabal (2000) ${ }^{[26]}$ used the nonlinear least square (NLS) method find the discharge history of the pollution source. They found the NLS was sensitive to observation error when the pollutant released gradually, but the discharge history could be identified with big observation error when the pollutant released abruptly.

\subsection{Direct methods}

Tikhonov regularization (TR) is the general method to convert the illposed problem to optimization of a well-posed problem. However, the feature of source function should be prior, and the regularization weight is very important to $\mathrm{PSI}{ }^{[10]}$.

Skaggs and Kabala (1994) ${ }^{[5]}$ applied TR on identifying a history of pollution plume and analyzed the influence of observation error, transport parameter and numerical stability. The observation error and the regularization weight had influence on the accuracy of PSI. Based on their research, Liu and Ball (1999) ${ }^{[10]}$ supposed the dispersion is the mental pollutant transport form, and evaluated plume history of trichloroethylene and tetrachloroethylene in the low permeable aquitard of Dover Air Force Base.

Neupauer, et al (2000)[27] compared the TR and Minimum Relative Entropy (MRE), and found that both the two methods could establish smooth function of source release history. MRE was better than TR, when the history was a piecewise function without error, but TR was more stable than MRE, when the error existed. However, the PSI solution quality was decided by the PSI problem and the subjective input of simulator [28]. Quasi-Reversibility (QR) was used to solve the invert dispersion problem, and was expanded to solve the convection-dispersion problem in a 1D aquifer by Skaggs and Kabala (1995)[29]. The TR was more accuracy than the $\mathrm{QR}$, and both of them had not been used in the PSI problem of heterogeneous aquifer ${ }^{[9]}$.

Adjoint method (AM) was numerical stable, and could reduce the number of simulation ${ }^{[4]}$. Neupauer and Wilson (1999) ${ }^{[30]}$ solved the 1D time-invert convection-dispersion equation. They showed that the backward model probabilities were adjoint states of resident concentration, and provided an approach for obtaining the governing equations and boundary and final conditions for these probabilities. Then they expanded their method into solving 3D time-invert convection-dispersion equation ${ }^{[31]}$.

\subsection{Probabilistic approaches}

Datta et al. (1989)[32] applied the statistical pattern recognition technique on the PSI, they studied the influence of parameter uncertainty, observation error and data missing on PSI. Their method could identify the pollution history when observation data was incomplete, and the approach required less data than the optimization approach to achieve comparable results ${ }^{[5]}$.

Wagner(1992) ${ }^{[33]}$ used the non-linear maximum likelihood estimation to determine optimal estimates of aquifer parameters and source characteristics based on measurements of hydraulic head and contaminant concentration. Contaminant flux at the source is estimated for a 2D groundwater system. The parameter distribution and source 
location were known, and the method was suitable for the pollution site with good observation data.

Bagtzoglou et al. ${ }^{[34]}$ applied the particle method to providing probabilistic estimates of source location and spill-time history in a 2D heterogeneous aquifer. The invert flow velocity field was used to simulated the reverse time, the convection was time-invert, but the dispersion was not. This method could be used for PSI problem when conductivity and distribution were well-known or uncertain.

Wilson and Liu (1994) ${ }^{[35]}$ solved the transport equation using stochastic differential equations backwards in time. They kept the dispersion part positive and invert the advection part. They claimed that by choosing the boundary condition properly, the method can be generalized to include linear adsorption with kinetic effects and 1st order decay. Then they expanded the method to a $2 \mathrm{D}$ heterogeneous aquifer [36], and the further study shown the travel time probability are in very close agreement with the simulation results from traditional forward-in-time methods.

Woodbury and Ulrych (1996) ${ }^{[37]}$ used the Minimum Relative Entropy (MRE) method to solve PSI problem. Based on the noise-free data, the method was able to reconstruct the plume evolution history indistinguishable from the true history. As for data with noise, the MRE managed to recover the salient features of the source history. Woodbury et al. (1998) extended the MRE to reconstruct a 3D plume source within a 1D constant velocity field and constant dispersivity system. The released history of 1,4-dioxane in the Gloucester Landfill was identified. They found that the early release history was not satisfied and long-time observation was very important for the identification of the whole release history.

Snodgrass and Kitanidis (1997) ${ }^{[38]}$ used Bayesian theory and geostatistical techniques to solve PSI problem. The source function to be estimated is discretized into components that are assigned a known stochastic structure with unknown stochastic parameters. No blind assumptions were needed for the nature and structure of the unknown source function. Limitation of this method is that the location of the potential source nust be known a priori. Michalak and Kitanidis (2004) ${ }^{[39]}$ used geostatistical approach to invers modeling. They recovered the antecedent distribution of a contaminant at a given point back in time. Sun (2007) ${ }^{[40]}$ used the robust geostatistical inversion approach to illustrated the source release history in a 2D aquifer. When the model is perfectly known, the solution of the method coincides with that of the geostatistical inversion approach. With the parameter and model uncertainty, this method gave a better performance than the geostatistical inversion approach did.

\section{Conclusion}

Groundwater pollution identification is the very first step for groundwater remediation. It is useful to decide the groundwater remediation strategy, and to determine the responsibility between different polluters. PSI is a difficult hotspot in hydrogeology, because it is a typical ill-posed problem which relates to different disciplines. Though many researchers have given great effort to extend the invert simulation method into more practical cases, most of the invert simulation methods were tested with ideal 1D or 2D numerical case, and these methods cannot solve actual PSI problem completely. Chemical reaction and microbial activity in groundwater are important factor for source identification, but a few researches involved the linear absorption process and simple organic pollutants in groundwater. So researchers have to keep trying their best to find practical methods to solve actual complex PSI problem. The more complex the PSI problem is, the more computational effort is consumed. To solve actual PSI problem, computational efficiency should be improved, especially for the optimization approaches. Some researchers studied the influence of the observation data and model parameter error on the PSI result, but model uncertainty is the intrinsic factor which could bring great influence on the PSI result. As a typical ill-posed problem, PSI is very sensitive to those uncertainty, so more work are going to be carried out to reduce the disturbance of these uncertainty.

\section{Acknowledgements}

The acknowledgement for the National Natural Science Foundation of China (Grants No. 51409161, No. 51509157), the Natural Science Foundation of Jiangsu Province (Grants No. BK20140080).

\section{References}

[1]Y. Q. Xue and Y. K. Zhang, "Twofold significance of ground water pollution prevention in China's water pollution control", Acta Scientiae Circumstantiae, 29, pp. 474-481, (2009). (in Chinese)
[2]J. J .Jiang, "Groundwater polltion and prevention of China", Environmental Protection, 38, pp. 16-17, (2007).(in Chinese)

[3]A. Sun, S. L. Painter, G. W. Wittmeyer. "A constrained robust least squares approach for contaminant source release history identification", Water Rescources Research, 42, W04414, doi:10.1029/2005WR004312, (2006).

[4]E. Milnes, P. Perrochet. "Simultaneous identification of a single pollution point-source location and contamination time under known flow field conditions", Advances in Water Resources, 30, pp. 2439-2446, (2007).

[5]T. H. Skaggs, Z. J. Kabala. "Recovering the release history of a groundwater contaminant", Water Resources Research, 30, pp.71-79, (1994).

[6]A. Woodbury, E. Sudicky, T. J. Ulrych, R.Ludwig. "Three-dimensional plume source Reconstruction using minimum relative entrop inversion", Journal of Contaminant Hydrology, 32, pp.131-158, (1998).

[7] S. N. Bashi-Azghadi, R. Kerachian, M. R. Bazargan-Lari, K. Solouki. "Characterizing and unknown pollution source in groundwater resources systems using PSVM and PNN", Expert Systems With Applications, 37, pp. 7154-7161, (2010).

[8]H. R. Ghafouri, B. S. Darabi. "Optimal identification of Ground-Water pollution sources", International Journal of Civil Engineering, 5, pp. 144154, (2007).

[9]J. Atmadja, A. C. Bagtzoglou. "State of the art report on mathematical methods for groundwater pollution source identification", Environmental Forensics, 2, pp. 205-214, (2001).

[10]C. Liu, W. P. Ball. "Application of inverse methods to contaminant source identification from aquitard diffusion profiles at Dover AFB Delaware", Water Resources Research, 35, pp. 1975-7985, (1999).

[11]A. C. Bagtzoglou, J. Atmadja. "Marching-jury backward beam equation and quasi-reversibility methods for hydrologic inversion: Application to contaminant plume spatial distribution recovery", Water Resources Research, 39, pp. 1038, doi:10.1029/2001WR001021, (2003).

[12]B. Y. Mirghani, K. G. Mahinthakumar, M. E. Tryby, R. S. Ranjithan, E. M. Zechman. "A parallel evolutionary strategy based simulation-optimization approach for solving groundwater source identification problems", Advances in Water Resources, 32, pp. 1373-1385, (2009).

[13]M. T. Ayvaz. "A linked simulation-optimization model for solving the unknown groundwater pollution source identification problems", Journal of Contaminant Hydrology, 117, pp. 46-59, (2010).

[14]S. M. Gorelick, B. Evans, I. Ramson. "Identifying sources of groundwater pollution: An optimization approach", Water Resources Research, 19, pp. 779-790, (1983).

[15]P. S. Mahar, B. Datta. "Optimal identification of ground-water pollution sources and parameter estimation", Journal of Water Resources Planning and Management, 127, pp. 20-29, (2001).

[16]B. A. Mirghani. "Evolutionary algorithms-based parallel simulationoptimization framework for solving inverse problems", Dissertation for Doctor of Pholosophy of North Carolina State University, (2007).

[17]M. M. Aral, J. Guan, M. L. Maslia. "Identification of contaminant source location and release history in aquifers", Journal of Hydrologic Engineering, 6, pp. 225-234, (2001).

[18]X. P. Fan, G. S. Li, "An improved genetic algorithm for groundwater pollution", Chinese Journal of Computational Physics, 24, pp. 187-191, (2007).(in Chinese)

[19]H. D. Yeh, T. Chang, Y. Liang. "Groundwater contamination source identification using hybrid optimization methods", Water Resources Research, 43, W09420, doi:10.1029/2005WR004731, (2007).

[20]T. V. Bharat, P. V. Sivapullaiah, M. M. Allam. “Swarm intelligence based inverse model for characterization of groundwater contaminant source", Electronic Journal of Geotechnical Engineering, 14, pp. 1-14, (2009).

[21]V. V. Vesselinov, D. R. Harp. "Adaptive hybrid optimization strategy for calibration and parameter estimation of physical process models", 
Computers \& Geosciences, 49, pp.10-20, (2010).

[22]R. M. Singh, B. Datta. "Groundwater pollution source identification and simultaneous parameter estimation using pattern matching by artificial neural network", Environmental Forensics, 5, pp. 143-159, (2004).

[23]N. K. Ala, P. A. Domenico. "Inverse analytical techniques applied to coincident contaminant distributions at Otis Air Force Base Massachusetts", Ground Water, 30, pp. 212-218, (1992).

[24]J. B. Butcher, T. D. Gauthier. "Estimation of residual dense NAPL mass by inverse modelling", Ground Water, 32, pp. 71-78, (1994).

[25]P. Sidauruk, A. Cheng, D. Ouazar. "Ground water contaminant source and transport parameter identification by correlation coefficient optimization", Ground Water, 36, pp. 208-214, (1998).

[26]S. Alapati, Z. J. Kabala. "Recovering the release history of a groundwater contaminant via the non-linear least-squares estimation", Hydrological Processes, 14, pp. 1003-1016, (2000).

[27]R. M. Neupauer, B. Borchers, J. L. Wilson. "Comparison of inverse methods for reconstructing the release history of a groundwater contamination source", Water Resources Research, 36, pp. 2469-2475, (2000).

[28]G. K. Mahinthakumar, M. Sayeed. "Hybrid genetic algorithm - local search methods for solving groundwater source identification inverse problems", Journal of Water Resources Planning and Management, 131, pp. 45-57, (2005).

[29]T. H. Skaggs, Z. J. Kabala. "Recovering the release history of a groundwater contaminant plume: method of quasi-reversibility", Water Resources Research, 30, pp. 71-79, (1995).

[30]R. M. Neupauer, J. L. Wilson. "Adjoint method for obtaining backwardin-time location and travel probabilities of a conservative groundwater contaminant", Water Resources Research, 35, pp. 3389-3398, (1999).

[31]R. M. Neupauer, J. L. Wilson. “Adjoint-derived location and travel time probabilities for a multi-dimensional groundwater system", Water
Resources Research, 37, pp. 1657-1668, (2001).

[32]B. Datta, J. E. Beegle, M. L. Kavvas. "Development of an expert system embedding pattern-recognition techniques for pollution-source identification, Technical Report: PB-90-185927/XAB, OSTI ID: 6855981", Department of Civil Engineering, California University, Davis, CA (USA), (1989).

[33]B. J. Wagner. "Simultaneous parameter estimation and contaminant source characterization for coupled groundwater flow and contaminant transport modelling", Journal of Hydrology, 135, pp. 275-303, (1992).

[34]A. C. Bagtzoglou, D. E. Dougherty, A. B. Tompson. "Application of particle methods to reliable identification of groundwater pollution sources", Water Resources Management, 6, pp. 15-23, (1992).

[35]J. L. Wilson, J. Liu. "Backward tracking to find the source of pollution", Waste Management From Risk to Remediation, 1, pp. 181-199, (1994).

[36]J. Liu, J. L. Wilson. "Modeling travel time and source location probabilities in two-dimensional heterogeneours aquifer", Proc. $5^{\text {th }}$ WERC Technology Development Conference, Las Cruces, New Mexico, pp. 59-76, (1995).

[37]A. D. Woodbury, T. J. Ulrych. "Minimum relative entropy inversion theory and application to recovering the release history of a groundwater contaminant", Water Resources Research, 32, pp. 2671-2681, (1996).

[38]M. F. Snodgrass, P. K. Kitanidis. "A geostatistical approach to contam inant source identification", Water Resources Research, 33, pp. 537-546, (1997).

[39]A. M. Michalak, P. K. Kitanidis. "Estimation of historical groundwater contaminant distributio $\mathrm{n}$ using the adjoint state method applied to geostatistical inverse modelling", Water Resources Research, 40, W08302, doi:10.1029/2004WR003214, (2004).

[40]A. Sun. "A robust geostatistical approach to contaminant source identification", Water Resources Research, 43 W02418,doi:10.1029/2006WR005106, (2007). 